\title{
Granular Cell Tumor of the Common Bile Duct: A Case Report of a Rare Anatomical Entity and Brief Review of the Literature
}

\author{
Anastasios Katsourakis $^{\mathrm{a}}$, Ioannis Dimitriou ${ }^{\mathrm{b}, \mathrm{e}}$, Efthimios Chatzitheoklitos ${ }^{\mathrm{c}}$, \\ Danai Chourmouzi ${ }^{\mathrm{d}}$, George Noussios ${ }^{\mathrm{b}}$
}

\begin{abstract}
Tumors of the biliary tree are usually malignant, with benign tumors of the biliary tree occurring rarely. A granular cell tumor (GCT) of the biliary tree is an extremely rare condition that is always benign, and effectively treated via surgery. A 30 -year-old Caucasian woman presented with a 3-month history of increasing pruritus and jaundice. The physical examination revealed mild abdominal pain in the left hypochondrium and the radiological examination revealed excessive dilation of the endohepatic and extrahepatic biliary trees, caused by an obstruction at the level of the distal common bile duct (CBD) and an ovoid, enhanced, solid mass located between the CBD, head of the pancreas, and second part of the duodenum. The patient underwent cholecystectomy and excision of the tumor and reconstruction was conducted via a Roux-en-Y hepaticojejunostomy. The microscopic examination and the immunohistochemical examination set the diagnosis of a GCT of the CBD. Thus far, only 82 cases of GCTs of the biliary tree have ever been reported in the English literature. Here we report an additional case of a GCT in the extrahepatic biliary tree (located at the CBD), and the first case ever reported in Greece. A GCT of the biliary tree is a rare condition that all physicians should be aware of when treating a patient with obstructive jaundice, and presenting with a tumor in the extrahepatic biliary tree.
\end{abstract}

Keywords: Granular cell tumor; Common bile duct; Biliary tract; Jaundice

Manuscript accepted for publication March 10, 2017

aDepartment of Surgery, "Agios Dimitrios" General Hospital of Thessaloniki, Thessaloniki, Greece

${ }^{b}$ Laboratory of Anatomy in the Department of Physical Education and Sports Medicine (Serres), Aristotele University of Thessaloniki, Thessaloniki, Greece 'Diavalkaniko Medical Centre, Thessaloniki, Greece

${ }^{d}$ Diagnostic Radiology Derpartment, Diavalkaniko Medical Centre, Thessaloniki, Greece

${ }^{\mathrm{e}}$ Corresponding Author: Ioannis Dimitriou, Laboratory of Anatomy in the Department of Physical Education and Sports Medicine (Serres), Aristotele University of Thessaloniki, Kanari 7 Stavroupoli, Thessaloniki 56430, Greece. Email: johndim577@yahoo.com

doi: https://doi.org/10.14740/jmc2783w

\section{Introduction}

Tumors of the biliary tree are usually malignant, with a poor prognosis, while benign tumors of the biliary tree are rare [1-6]. Granular cell tumors (GCTs) of the biliary tree are extremely rare, and according to the literature, are always benign $[1-4,7-10]$. GCTs are usually located in the skin, subcutaneous tissues, oral cavity, head, and neck $[2,7,8,11]$. They are infrequently located in the gastrointestinal tract, and rarely located in the biliary tree $(<1 \%)[2,3,7,9,11]$. GCTs are related to the peripheral nerves, and it is generally accepted that they have a neurogenic origin and arise from Schwann cells $[1-5$, 7-11]. Thus far, few cases of GCTs in the extrahepatic biliary tree have been reported, but no author has ever reported a malignant GCT in the biliary system [1-5, 7-11]. GCTs of the biliary tree are usually found in young African-American females between 30 and 40 years of age [1-3, 7-10]. The symptoms are mild, the radiological findings are non-specific, and the diagnosis is made postoperatively, based on the pathological and histochemical findings [1-3, 7-10]. Surgery is often radi$\mathrm{cal}$, with these patients undergoing major procedures due to the suspicion of malignancy in the biliary tree [1-3, 7-10]. Almost all of the cases that have been reported had good prognoses, with the mortality and morbidity being caused by reasons other than the tumor's biology $[1,2,10]$.

\section{Case Report}

A 30-year-old Caucasian woman presented to our clinic with a 3-month history of increasing pruritus and jaundice, and did not complain of any other symptoms. Her medical history was free from any underlying disease, and she was not taking any medication. Her family history was unremarkable, and her gynecological history revealed one natural delivery. Overall, the patient was in good physical condition and completely fit. The physical examination revealed mild abdominal pain in the left hypochondrium that did not cause any discomfort to the patient, with no signs from any of the other systems. Her vital signs were normal, and the laboratory results were all within the normal ranges, except for mild transaminasemia and small elevations in the total and conjugated bilirubin levels.

The patient underwent computed tomography (CT), which 

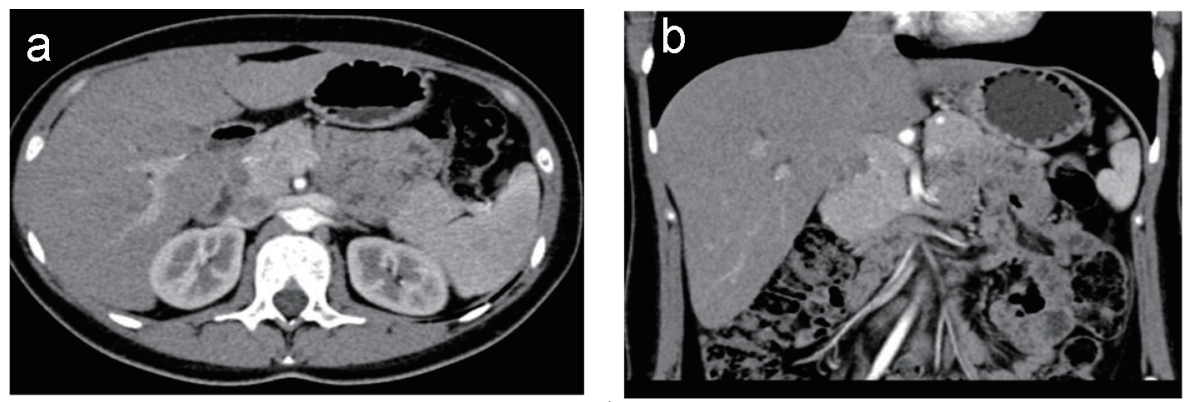

Figure 1. (a) Axial CT and (b) coronal reconstruction images show that there is no any visible mass.
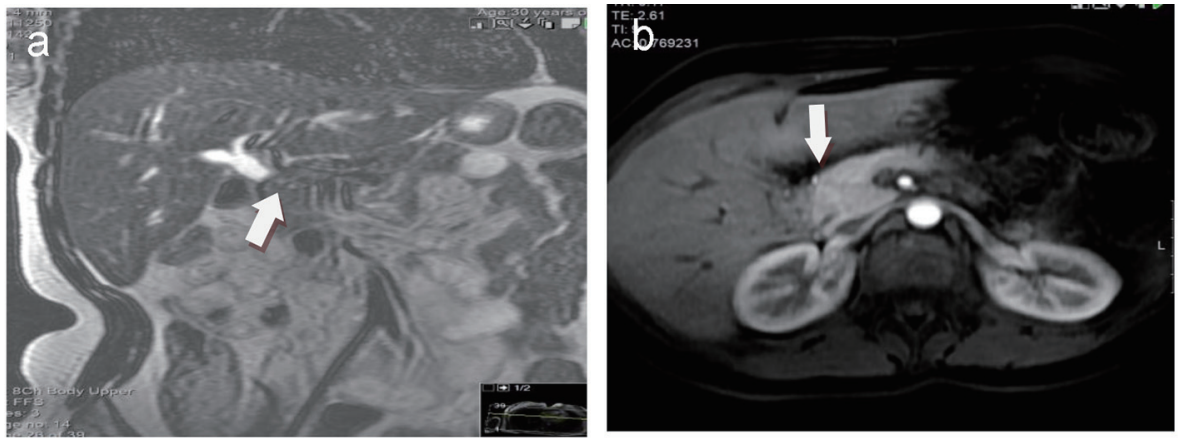

Figure 2. MRI coronal T2-weighted image (a) and axial post-contrast vibe image (b) show dilatation of the common bile duct and normal appearance of pancreatic head.

revealed excessive dilation of the endohepatic and extrahepatic biliary trees, caused by an obstruction at the level of the distal common bile duct (CBD) without revealing any visual mass (Fig. 1). The CBD below this level was stenotic, while the pancreas, pancreatic duct, and all of the other structures were normal. This patient then underwent magnetic resonance imaging (MRI) (Fig. 2) and magnetic resonance cholangiopancreatography (MRCP) which revealed an ovoid, enhanced, solid mass $13 \mathrm{~mm}$ in diameter located between the CBD, head of the pancreas, and second part of the duodenum (Fig. 3). This mass caused severe obstruction of the CBD lumen, which was proximally dilated, as well as the common hepatic duct (11 $\mathrm{mm}$ ) and the portal endohepatic bile ducts (about $9 \mathrm{~mm}$ ). All of the other anatomical structures were found to be normal. Pathologically enlarged lymph nodes were not observed in the

\section{CT scan or MRI-MRCP.}

After the examination, the patient was scheduled for surgery with the diagnosis of a possible cholangiocarcinoma of the lower third of the CBD, and a cholecystectomy was performed via open laparotomy. During the surgery, a small tumor was found in the lower third of the CBD, which was excised, and reconstruction was conducted via a Roux-en-Y hepaticojejunostomy. The patient's recovery was fast and uncomplicated, and she was discharged from the hospital on the sixth postoperative day.

The gross examination revealed an intramural, yellow, oval-shaped solid tumor that extended inside the CBD lumen, causing an obstruction, which was $15 \mathrm{~mm}$ at its largest diameter. This mass was located $3 \mathrm{~mm}$ centrally from the distal end of the CBD, above the pancreas. A GCT of the CBD was shown
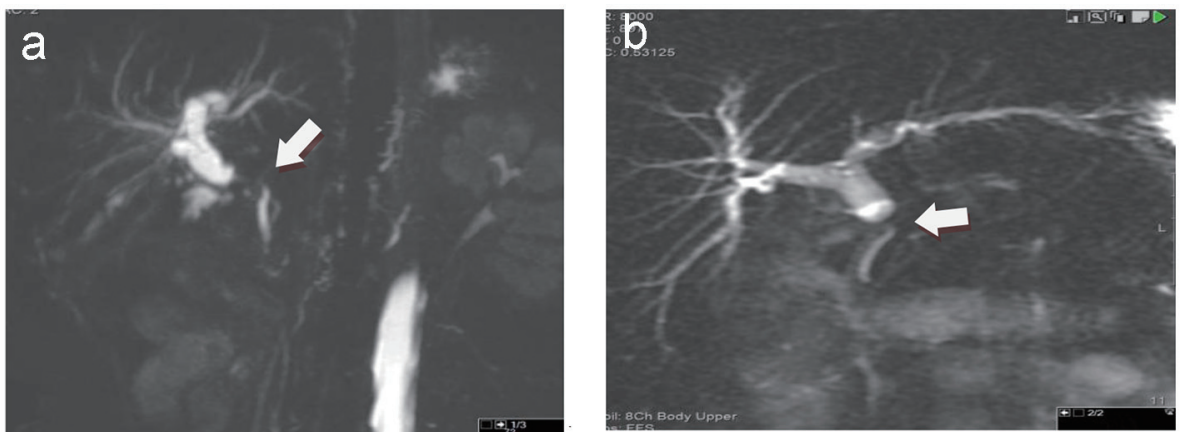

Figure 3. On MRCP, 2D (a) and 3D (b) dilatation of the intrahepatic bile ducts as well as dilatation of the common bile duct with ill-defined intraluminal filling defect is depicted. The distal common bile duct is normal. 

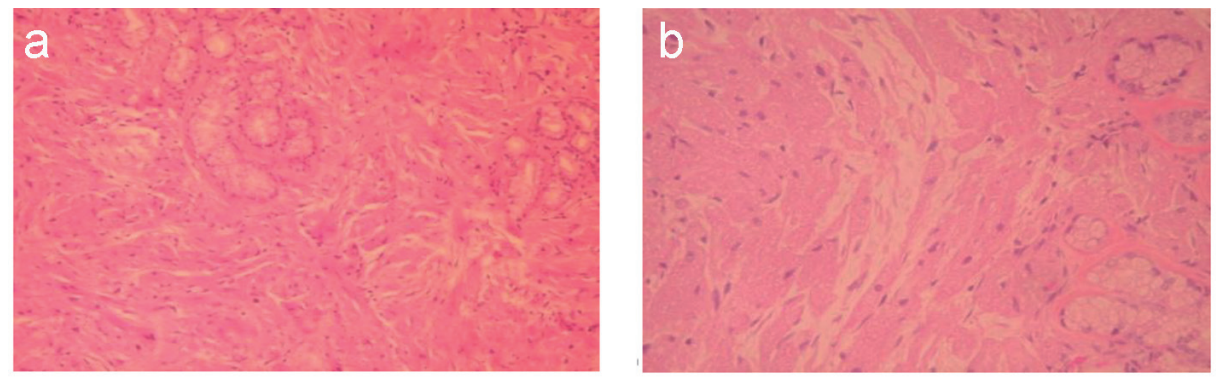

Figure 4. Histological examination shows infiltration around epithelial elements of ampulla of Vater by large polygonal cells with eosinophilic granular cytoplasm and small round regular usually eccentric nucleus $(\mathrm{H} \& \mathrm{E}, \times 100)(\mathrm{a})$. The same image in higher magnification $(H \& E, \times 200)(b)$

by the histological examination. The microscopic examination showed a well-demarcated mass consisting of semicircular, ovoid, and slightly elongated cells, with rich eosinophilic and granular cytoplasm, containing small semicircular, ovoid, and slightly elongated mild hyperchromatic nuclei. The cells were located in nests and cords separated by fibrous bands (Fig. 4). Necrosis and mitotic activity were not observed in the specimen, and invasion of the tumor capsule was not discovered in any of the tumor specimens. Small groups of glandular cells were observed between the granular cell nests, and were composed of cylindrical epithelium without atypia (Fig. 4a, b). The adjacent tissues were found to be completely normal. The immunohistochemical examination revealed that the tumor cells were positive for neurospecific enolase (NSE) and CD68, and strongly positive for the S-100 protein (Fig. 5). The pathological examination of the gall bladder showed chronic hyperplastic cholecystitis.

Considering the abovementioned findings, the tumor was diagnosed as a GCT of the CBD. The patient was followed up postoperatively, and found to have recovered completely. After 15 months, the patient was well and disease-free.

\section{Discussion}

A GCT was first described by Abrikossoff in 1926, and the first similar tumor in the biliary tree was described by Coggins in 1952 [1-4, 7-10]. Since then, many authors have reported GCTs, and thus far, 82 cases of GCTs in the biliary tree have been reported in the English literature [8]. Here we report the 83rd case of a GCT in the extrahepatic biliary tree (located in the $\mathrm{CBD}$ ), and the first case ever reported in Greece.

GCTs are rare benign tumors that usually appear in African-American women during the third and fourth decades of life [1-4, 7-10]. The mean time of appearance is 31 years of age, and ranges from 14 to 91 years of age [1]. The ratio between female and male occurrences is almost 4:1, taking into account all of the cases reported with GCTs of the biliary system [2].

These tumors are exclusively diagnosed after pathological and immunohistochemical examinations, which suggest a strong correlation between these tumors and peripheral nerves $[1-4,7-10]$. After many reports and discussions, it is now generally accepted that GCTs arise from Schwann cells, which is confirmed by the expression of the S-100 protein in the immunohistochemical analyses [1-5, 7-9].

Clinically, when GCTs develop in the biliary tree, the patients appear with symptoms such as abdominal pain, biliary colic, and obstructive jaundice, but these symptoms are nonspecific and can lead to many diagnoses $[1,7,11]$. The jaundice worsens over time and can be accompanied by pruritus [7]. GCTs are usually located in the skin, subcutaneous tissues, head, neck, and chest $[2,7,8]$. They have also been reported in the breast, larynx, uterus, intestine, esophagus, vocal cords, bronchus, and practically all other parts of the human body [3, $4,8,9]$. Their location in the gastrointestinal system is rare, ranging between $5 \%$ and $9 \%$, but in the biliary tree the presence is less than $1 \%[3,8,9]$. The preoperative diagnosis of
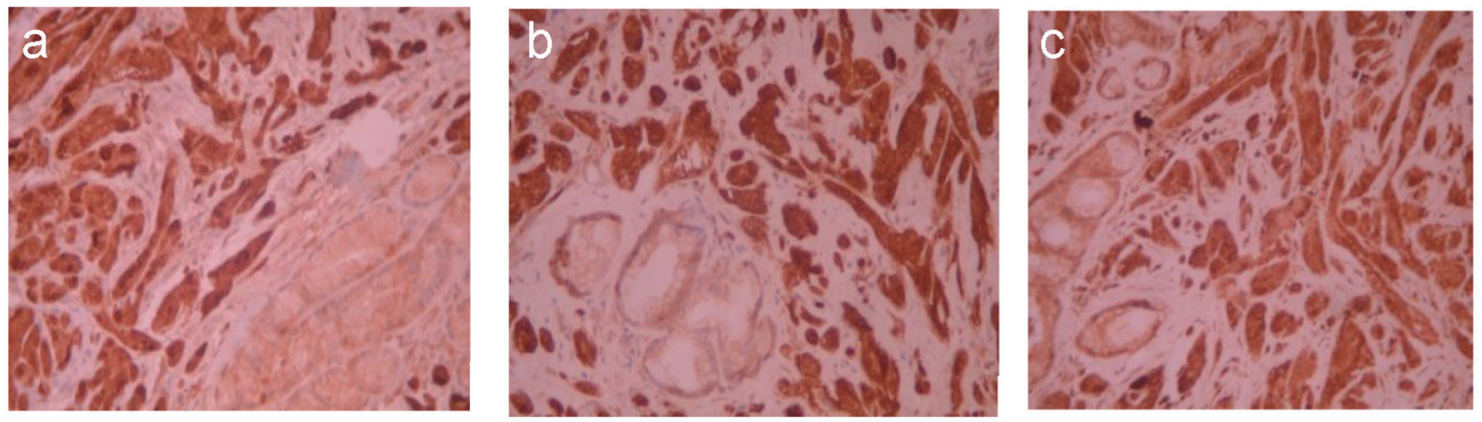

Figure 5. Immunohistochemical examination shows that tumor cells are positive for S-100 protein (immunostain, $\times 200)(a)$, CD68 (immunostain, $\times 200)($ b), and NSE (immunostain, $\times 200)(c)$. 
a GCT in the biliary tree is almost impossible, and is usually made after the surgery and the pathological, anatomical, and histochemical analyses of the biopsy of the excised specimen $[3,9]$. When GCTs are superficially located, a biopsy and diagnosis are easily conducted, but these are very difficult when the tumors invade and develop in the biliary tree [3]. The differential diagnosis of a GCT in the biliary tree includes malignant tumors like cholangiocarcinoma and adenocarcinoma, and benign diseases like sclerosing cholangitis, adenomas, papillomas of the extrahepatic biliary system, postoperative stenosis, and choledochocele $[1,3,7]$. Following the excision of the tumor, many biopsies are usually required to confirm the diagnosis [2]. According to the literature, only two cases of GCTs of the biliary tree have been diagnosed preoperatively thus far [10]. Both diagnoses were made after biopsies that were taken during endoscopic retrograde cholangiopancreatography (ERCP) procedures [10]. One alternative diagnostic modality that has been proposed for these tumors is an intraoperative needle biopsy and frozen section $[10,11]$. An ideal goal is to achieve treatment while avoiding major surgery, using ERCP as a diagnostic tool to take many deep biopsies from the CBD and common hepatic duct $[10,11]$.

Radiologically, a GCT of the biliary tree appears as a soft tissue mass that causes obstruction from outside or from within the lumen of the CBD or the hepatic ducts [7]. CT imaging shows an isodense tumor and MRI shows an isointense tumor of the hepatic parenchyma, which enhances after the injection of a contrast agent [7]. MRCP reveals the stenosis, and an endoscopic ultrasound shows a hypoechoic mass with welldefined borders [7]. A gross examination shows that GCTs are solitary, yellowish-white, oval shaped, painless nodules of less than $3 \mathrm{~cm}$ in their maximum diameter [1]. A microscopic examination of these tumors reveals the presence of polygonal or ovoid to round cells with eosinophilic granular cytoplasm, and small regular uniform hyperchromatic nuclei [1-3]. The cells are arranged in nests and cords, appear as clusters or sheets, and infiltrate the tissues separated by thin fibroconnective tissue septa $[1,3]$. Mitoses are absent, and there is no atypia or necrosis $[3,8]$. Immunohistochemical analyses indicate a non-epithelial origin with characteristics of neuroectodermal (Schwannian) origin [1-4, 9]. An examination using electron microscopy reveals cytoplasmic lysosomes filled with lipid material and microtubules that are related to myelin, characteristics that confirm the diagnosis of a GCT [3]. All GCT cells show strong positivity for the $\mathrm{S}-100$ protein, but negative results for some other staining [2-4, 7, 8].

The standard treatment goal for a GCT is excision of the tumor, with free tumor margins, and restoration of the bilioenteric continuity $[1-3,7-9,11]$. A Roux-en-Y hepaticojejunostomy is the best choice in this case [8-10]. When the intrapancreatic part of the CBD is involved, the recommended solution is a Whipple pancreatoduodenectomy, even though diversions and simple bypasses have been reported for tumors in this location $[10,11]$. The treatment of these tumors with stents using an endoscopic or percutaneous approach is unacceptable, and not appropriate for this curable disease $[1-3,7,9]$. Generally, the extent of the excision depends on the tumor's location and size $[7,11]$. If the tumor is located at the bile cyst or the cystic duct, then all that is needed is a simple cholecystectomy with free tumor margins $[9,11]$. In those cases in which a benign tumor is suspected in the biliary tree, it is not easy to exclude malignancy due its rarity, and major surgery may still be necessary for treatment $[2,6,10]$. If a biliary obstruction persists over time and is left untreated, secondary biliary cirrhosis will appear and may cause liver failure requiring liver transplantation $[3,7]$. Chemotherapy and radiotherapy have no effect in the treatment of these tumors $[10,11]$.

Long-term follow-up is necessary $[3,11]$. Based on the literature, only one patient died after the excision of a GCT due to other medical reasons [2] and only two patients (of the 83 ) had recurrence due to incomplete excision $[1,2,5,10,11]$. Thus far, there has not been a single case referring to a malignant GCT in the biliary tree, even though they have been reported in other parts of the human body [9]. There have been five cases of multifocal GCTs and five cases of concomitant extrabiliary involvement reported in the literature $[1,9]$. Moreover, the prognosis is excellent after a free margin excision [1, $3,10]$. Overall, morbidity depends on the surgery, and there is no mortality based on the nature of the tumor [2].

In conclusion, a GCT of the biliary tree is a rare condition that all physicians should be aware of when treating a patient with obstructive jaundice, and presenting with a tumor in the extrahepatic biliary tree. Although rare, GCTs should be included in the differential diagnosis of tumors that cause obstruction of the biliary tree [2]. Local excision of the tumor and reconstruction of bilioenteric continuity is the ideal treatment [1-3, 7-9], but demands preoperative suspicion in order to avoid major surgery, as long as biliary tumors are considered to be malignant [7]. Wide resections offer the best chance for a cure, and there must be a low threshold for an extensive resection when facing tumors in the biliary tree that cannot be easily diagnosed preoperatively, such as GCTs $[10,11]$.

\section{Competing Interests}

The author(s) declare that they have no competing interests.

\section{Consent}

Written informed consent was obtained from the patient for publication of this case report and accompanying images.

\section{References}

1. te Boekhorst DS, Gerhards MF, van Gulik TM, Gouma DJ. Granular cell tumor at the hepatic duct confluence mimicking Klatskin tumor. A report of two cases and a review of the literature. Dig Surg. 2000;17(3):299-303.

2. Saito J, Kitagawa M, Kusanagi H, Kano N, Ishii E, Nakaji S, Hirata N, et al. Granular cell tumor of the common bile duct: a Japanese case. World J Gastroenterol. 2012;18(43):6324-6327.

3. Patel AJ, Jakate SM. Granular cell tumor of the biliary tract. Gastroenterol Hepatol (N Y). 2010;6(5):331-336. 
4. Cheslyn-Curtis S, Russell RC, Rode J, Dhillon AP. Granular cell tumour of the common bile duct. Postgrad Med J. 1986;62(732):961-963.

5. Lewis WD, Buell JF, Jenkins RL, Burke PA. Biliary duct granular cell tumor: a rare but surgically curable benign tumor. HPB Surg. 1993;6(4):311-317.

6. Chamberlain RS, Blumgart LH. Carcinoid tumors of the extrahepatic bile duct. A rare cause of malignant biliary obstruction. Cancer. 1999;86(10):1959-1965.

7. Kamaoui I, Pilleul F. Granular cell tumour of the common bile duct: a condition to be aware of. Diagn Interv Imaging. 2013;94(3):324-326.

8. Jung C, Heo I, Kim SB, Park S, Cho SY. Biliary granular cell tumor. J Pathol Transl Med. 2015;49(1):89-91.

9. Bilanovic D, Boricic I, Zdravkovic D, Randjelovic T, Stanisavljevic N, Tokovic B. Granular cell tumor of the common hepatic duct presenting as cholangiocarcinoma and acute acalculous cholecystitis. Acta Chir Iugosl. 2008;55(4):99-101.

10. Tonsi A, Standish R, Desai C, Davidson BR. Granular cell tumour of the bile duct mimicking distal cholangiocarcinoma: one case report and review of the literature. Minerva Chir. 2006;61(3):247-255.

11. Khalid K, Alam MK, Al-Shakweer WA, Al-Teimi IN. Granular cell tumour of the ampulla of Vater. J Postgrad Med. 2005;51(1):36-38. 